\title{
Energy Efficient Scheme for Clustering Protocol Prolonging the Lifetime of Heterogeneous Wireless Sensor Networks
}

\author{
Parul Saini \\ Department of Computer Science \& Engineering \\ National Institute of Technology Jalandhar, \\ Punjab, India
}

\author{
Ajay K Sharma \\ Department of Computer Science \& Engineering \\ National Institute of Technology Jalandhar, \\ Punjab, India
}

\begin{abstract}
In recent advances, many routing protocols have been proposed based on heterogeneity with main research goals such as achieving the energy efficiency, lifetime, deployment of nodes, fault tolerance, latency, in short high reliability and robustness. In this paper, we have proposed an energy efficient cluster head scheme, for heterogeneous wireless sensor networks, by modifying the threshold value of a node based on which it decides to be a cluster head or not, called TDEEC (Threshold Distributed Energy Efficient Clustering) protocol. Simulation results show that proposed algorithm performs better as compared to others.
\end{abstract}

\section{Keywords}

Cluster head, residual energy, heterogeneity, energy efficient, wireless sensor networks.

\section{INTRODUCTION}

Wireless sensor networks composed of hundreds of sensor nodes which sense the physical environment in terms of temperature, humidity, light, sound, vibration, etc. The main task of sensor node is to gather the data and information from the sensing field and send this to the end user via base station. These sensor nodes can be deployed on many applications. Current wireless sensor network is working on the problems of low-power communication, sensing, energy storage, and computation.

Clustering technique enables the sensor network to work more efficiently. It increases the energy consumption of the sensor network and hence the lifetime [1]. The main role of cluster head is to provide data communication between sensor nodes and the base station efficiently. So the cluster head should have high energy as compared to other nodes, also, it performs the data aggregation.

LEACH by Heinzelman, et. al. [2] is the first hierarchical or clustering-based protocol in which cluster heads are randomly selected. Others advancements proposed for LEACH are LEACHC [3], HEED [4], SEP [5], ALEACH [6].

DEEC [7] is cluster-based algorithm in which cluster heads are selected on the basis of probability of ratio of residual energy and average energy of the network. In this algorithm, node having more energy has more chances to be a cluster head. It prolongs the lifetime of the network. In this paper our proposed scheme is TDEEC (Threshold Distributed Energy Efficient Clustering) scheme which follows the thoughts of DEEC. This scheme selects the cluster heads from the high energy nodes improving energy efficiency and lifetime of the network.
The rest of the paper is organized as follows: Section 2 contains the related work done. Section 3 explains the radio energy dissipation model and network model with its assumptions followed by section 4 which describes our approach. Section 5 shows the simulation results followed by conclusion and references.

\section{RELATED WORK}

Heinzelman, et. al. [2] introduced a hierarchical clustering algorithm for sensor networks, called Low Energy Adaptive Clustering Hierarchy (LEACH) for homogeneous wireless sensors networks. LEACH is a cluster-based protocol, which includes distributed cluster formation. LEACH randomly selects a few sensor nodes as cluster heads (CHs) and rotates this role to evenly distribute the energy load among the sensors in the network [1]. PEGASIS [8] is a chain based protocol which avoids cluster formation and uses only one node in a chain to transmit to the BS instead of using multiple nodes.

Manjeshwar et. al. proposed Threshold sensitive Energy Efficient sensor Network protocol (TEEN) [9]. TEEN pursues a hierarchical approach along with the use of a data-centric mechanism. In TEEN, the cluster head broadcasts two thresholds to the nodes. These are hard and soft thresholds for sensed attributes. TEEN is not good for applications where periodic reports are needed since the user may not get any data at all if the thresholds are not reached. Manjeshwar et. al. then proposed Adaptive Threshold sensitive Energy Efficient sensor Network protocol (APTEEN) [10] which aims at both capturing periodic data collections and reacting to time-critical events. The architecture is same as in TEEN. The main drawbacks of TEEN and APTEEN are the overhead and complexity of forming clusters in multiple levels implementing threshold-based functions and dealing with attribute-based naming of queries.

Heinzelman, et.al. [3] proposed LEACH-centralized (LEACH-C), a protocol that uses a centralized clustering algorithm and the same steady-state protocol as LEACH. O. Younis, et.al [4] proposed HEED (Hybrid Energy-Efficient Distributed clustering), which periodically select cluster heads according to a hybrid of the node residual energy and a secondary parameter, such as node proximity to its neighbors or node degree. G. Smaragdakis, I. Matta, A. Bestavros proposed SEP (Stable Election Protocol) [5] in which every sensor node in a heterogeneous two-level hierarchical network independently elects itself as a cluster head based on its initial energy relative to that of other nodes. Li Qing et.al. [7] Proposed DEEC (Distributed energy efficient Clustering) algorithm in which cluster head is selected on the basis of 
probability of ratio of residual energy and average energy of the network. Simulations show that its performance is better than other protocols.

Md. Solaiman Ali, et.al [6] proposed ALEACH (Advanced LEACH) a new technique to select the cluster heads in every round which depends both on current state probability and general probability. Sajjanhar et al. [11] proposed a Distributive Energy Efficient Adaptive Clustering (DEEAC) Protocol, which is having spatio-temporal variations in data reporting rates across different regions. DEEAC selects a node to be a cluster head depending upon its hotness value and residual energy.

B. Elbhiri et al [12], proposed SDEEC (Stochastic Distributed Energy-Efficient Clustering (SDEEC) SDEEC introduces a balanced and dynamic method where the cluster head election probability is more efficient. Moreover, it uses a stochastic scheme detection to extend the network lifetime. Simulation results show that this protocol performs better than the Stable Election Protocol (SEP) and the Distributed Energy- Efficient Clustering (DEEC) in terms of network lifetime.

Inbo Sim, et.al [13] proposed ECS (Energy efficient Cluster header Selection) algorithm which selects $\mathrm{CH}$ by utilizing only its information to extend network lifetime and minimize additional overheads in energy limited sensor networks. Ma Chaw Mon Thein, et.al [14] proposed a modification of the LEACH's stochastic cluster-head selection algorithm by considering the additional parameters, the residual energy of a node relative to the residual energy of the network for adapting clusters and rotating cluster head positions to evenly distribute the energy load among all the nodes.

We have proposed an approach called threshold distributed energy efficient clustering (TDEEC) algorithm whose main aim is to increase the energy efficiency and stability of the heterogeneous wireless sensor networks.

\section{RADIO ENERGY DISSIPATION MODEL AND NETWORK MODEL \\ 3.1 Radio Energy Dissipation Model}

Radio Energy Model used is based on [2,3]. Energy model for the radio hardware energy dissipation where the transmitter dissipates energy to run the radio electronics and the power amplifier, and the receiver dissipates energy to run the radio electronics is shown in Figure 1 [2, 3].

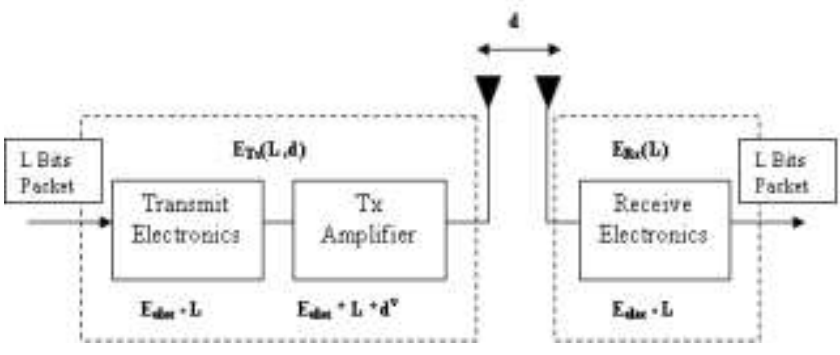

Figure 1 Radio Energy Dissipation Model

In this model, both the free space ( $\mathrm{d}^{2}$ power loss) and the multipath fading $\left(\mathrm{d}^{4}\right.$ power loss) channel models were used, depending on the distance between the transmitter and receiver [2, 9]. Power control can be used to invert this loss by appropriately setting the power amplifier-if the distance is less than a threshold $\mathrm{d}_{0}$, the free space model is used; otherwise, the multipath model is used. Thus, to transmit an L-bit message a distance, the radio expands.

$$
E_{T x}(L, d)= \begin{cases}L \cdot E_{\text {elec }}+L \cdot E_{f s} \cdot d^{2} & \text { if } d<d_{o} \\ L \cdot E_{e l e c}+L \cdot E_{a m p} \cdot d^{4} & \text { if } d \geq d_{o}\end{cases}
$$

The electronics energy, $\mathrm{E}_{\text {elec }}$, depends on factors such as the digital coding, modulation, filtering, and spreading of the signal, whereas the amplifier energy, $\mathrm{E}_{\mathrm{fs}} \cdot \mathrm{d}^{2}$ or $\mathrm{E}_{\mathrm{amp}} \cdot \mathrm{d}^{4}$, depends on the distance to the receiver and the acceptable bit-error rate $[2,3]$.

Value of threshold distance $d_{o}$ is given by

$$
\mathrm{d}_{\mathrm{o}}=\frac{\mathrm{E}_{\mathrm{fs}}}{\mathrm{E}_{\mathrm{amp}}}
$$

\subsection{Network Model}

Network model used consists of N nodes in M X M network field as shown in Figure 2.

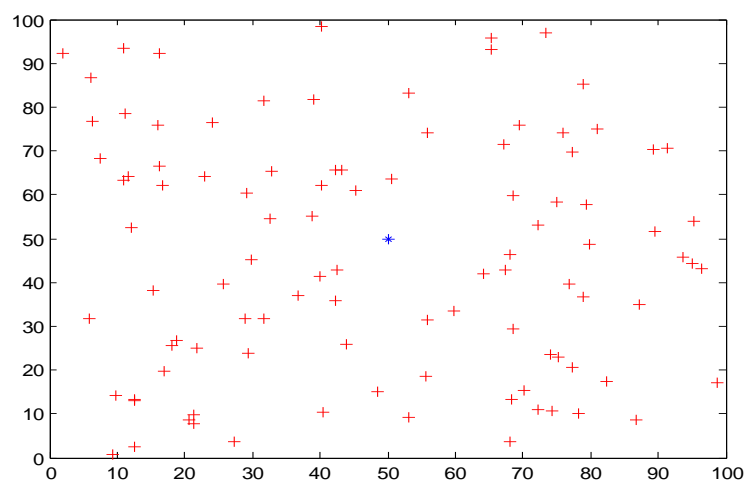

Figure 2 Random Wireless Sensor Model

In the network model some assumptions have been made for the sensor nodes as well as for the network. Hence the assumptions and properties of the network and sensor nodes are:

- Sensor Nodes are uniformly randomly deployed in the network.

- There is one Base Station which is located at the centre of the sensing field.

- Nodes always have the data to send to the base station.

- Nodes are location-unaware, i.e. not equipped with GPScapable antennae.

- All nodes have similar capabilities in terms of processing and communication and of equal significance. This motivates the need for extending the lifetime of every sensor.

- Sensor nodes have heterogeneity in terms of energy i.e., different energy levels. All nodes have different initial energy; some nodes are equipped with more energy than the normal nodes

\subsubsection{Two-level heterogeneous networks}

We have used two types of nodes in the network, normal and advanced nodes. $E_{o}$ is the initial energy of the normal nodes, and $\mathrm{m}$ is the fraction of the advanced nodes, which own a times more energy than the normal ones. Thus there are m.N advanced nodes equipped with initial energy of Eo $(1+a)$, and $(1-m) . N$ normal nodes that are equipped with initial energy of $E_{o}$. The total initial energy of the two-level heterogeneous networks is given by: 


$$
\begin{aligned}
\mathrm{E}_{\text {total }} & =\mathrm{N} \cdot(1-\mathrm{m}) \cdot \mathrm{E}_{\mathrm{o}}+\mathrm{m} \cdot \mathrm{N} \cdot(1+\mathrm{a}) \cdot \mathrm{E}_{\mathrm{o}} \\
& =\mathrm{N} \cdot \mathrm{E}_{\mathrm{o}} \cdot(1+\mathrm{a} \cdot \mathrm{m})
\end{aligned}
$$

Therefore, the two-level heterogeneous networks have a.m times more energy and virtually a.m more nodes [7].

\subsubsection{Three-level heterogeneous networks}

In three-level heterogeneous networks, there are three types of sensor nodes $[15,16]$. They are normal nodes, advanced nodes and super nodes. Let $\mathrm{m}$ be the fraction of the total number of nodes $\mathrm{N}$, and mo is the percentage of the total number of nodes which are equipped with $\mathrm{b}$ times more energy than the normal nodes, called as super nodes, the number is N.m.mo. The rest N. $\mathrm{m}$. (1-mo) nodes are equipped with a times more energy than the normal nodes; called as advanced nodes and remaining N. (1-m) as normal nodes. The total initial energy of the three-level heterogeneous networks is given by:

$$
\begin{aligned}
\mathrm{E}_{\text {total }}= & \mathrm{N} \cdot(1-\mathrm{m}) \cdot \mathrm{E}_{\mathrm{o}}+\mathrm{N} \cdot \mathrm{m} \cdot(1-\mathrm{mo}) \cdot(1+\mathrm{a}) \cdot \mathrm{E}_{\mathrm{o}} \\
& +\mathrm{N} \cdot \mathrm{m} \cdot \mathrm{mo} \cdot \mathrm{E}_{\mathrm{o}} \cdot(1+\mathrm{b}) \\
= & N \cdot E_{o} \cdot(1+m \cdot(a+m o \cdot b))
\end{aligned}
$$

Therefore, the three-level heterogeneous networks have $\mathrm{m}$. $(\mathrm{a}+$ mo. b) times more energy or we can say that the total energy of the system is increased by a factor of $(1+\mathrm{m}$. $(\mathrm{a}+\mathrm{mo} . \mathrm{b}))[15$, $16]$.

\subsubsection{Multilevel heterogeneous networks}

In multi-level heterogeneous networks, initial energy of sensor nodes is randomly distributed over the close set $\left[\mathrm{E}_{0}, \mathrm{E}_{\mathrm{o}}(1+\right.$ $\left.a_{\max }\right)$ ], where $E_{o}$ is the lower bound and $a_{\max }$ determine the value of the maximal energy. Initially, the node $s_{i}$ is equipped with initial energy of $E_{o} .\left(1+a_{i}\right)$, which is $a_{i}$ times more energy than the lower bound $\mathrm{E}_{\mathrm{o}}$. The total initial energy of the multi-level heterogeneous networks is given by [7]:

$$
\mathrm{E}_{\text {total }}=\sum_{\mathrm{i}=1}^{\mathrm{N}} \mathrm{Eo}\left(1+\mathrm{a}_{\mathrm{i}}\right)=\mathrm{Eo}\left(\mathrm{N}+\sum_{\mathrm{i}=1}^{\mathrm{N}} \mathrm{a}_{\mathrm{i}}\right)
$$

\section{TDEEC}

TDEEC implements the same strategy for estimating the energy in the network as proposed in DEEC [7]. Since the probabilities calculated depend on the average energy of the network at round $r$, hence the average energy is estimated as:

$$
\bar{E}(r)=\frac{1}{N} E_{\text {total }}\left(1-\frac{r}{R}\right)
$$

where $\mathrm{R}$ denotes the total rounds of the network lifetime. $\mathrm{R}$ can be calculated as

$$
R=\frac{E_{\text {total }}}{E_{\text {round }}}
$$

$\mathrm{E}_{\text {round }}$ is the energy dissipated in the network in a round.

The total energy dissipated $\mathrm{E}_{\text {round }}$ is equal to

$$
\mathrm{E}_{\text {round }}=\mathrm{L}\left(2 \mathrm{NE}_{\text {elec }}+\mathrm{NE}_{\mathrm{DA}}+\mathrm{kE}_{\mathrm{amp}} \mathrm{d}_{\mathrm{toBS}}^{4}+\mathrm{NE}_{\mathrm{fs}} \mathrm{d}_{\mathrm{toCH}}^{2}\right)
$$

where $\mathrm{k}$ is number of clusters $\mathrm{d}_{\mathrm{toBS}}$ is the average distance between cluster head and the base station and $\mathrm{d}_{\mathrm{toCH}}$ is the average distance between the cluster members and the cluster head.

$$
\text { Now, } \quad d_{\text {toCH }}=\frac{M}{\sqrt{2 \pi k}} d_{\text {toBs }}=0.765 \frac{\mathrm{M}}{2}
$$

By calculating the derivative of $E_{\text {round }}$ with respect to $k$ to zero we get optimal number of clusters as

$$
\mathrm{k}_{\mathrm{opt}}=\sqrt{\frac{\mathrm{N}}{2 \pi}} \frac{\mathrm{M}}{\mathrm{d}_{\mathrm{toBs}}^{2}} \sqrt{\frac{\mathrm{E}_{\mathrm{fs}}}{\mathrm{E}_{\mathrm{amp}}}}
$$

Hence we can find the energy dissipated per round by substituting equations (9) \& (10) in (8).Due to the heterogeneity factors $\mathrm{R}$ is taken as $1.5 \mathrm{R}$ (Since $\overline{\mathrm{E}}(\mathrm{r}$ ) will be too large at the end from Eq.(6), some will not die finally).

Traditionally as per LEACH, Cluster head algorithm is broken into rounds. At each round node decides whether to become a cluster head based on threshold calculated by the suggested percentage of cluster heads for the network (determined a priori) and the number of times the node has been a cluster-head so far. This decision is made by the nodes by choosing the random number between 0 and 1 . If the number is less than a threshold $\mathrm{T}$ (s) the node becomes a cluster-head for the current round. The threshold is set as:

$$
T(s)=\left\{\begin{array}{cl}
\frac{p}{1-p \cdot\left(r \bmod \frac{1}{p}\right)} & \text { if } s \in G \\
0 & \text { Otherwise }
\end{array}\right.
$$

where $\mathrm{p}, \mathrm{r}$, and $\mathrm{G}$ represent, respectively, the desired percentage of cluster-heads, the current round number, and the set of nodes that have not been cluster-heads in the last $1 / \mathrm{p}$ rounds. Using this threshold, each node will be a cluster head, just once at some point within $1 / \mathrm{p}$ rounds.

In TDEEC approach we have adjusted the value of the threshold, according to which a node decides to be a cluster head or not, based on ratio of residual energy and average energy of that round in respect to the optimum number of cluster heads. So that only nodes having a more energy becomes the cluster head.

The threshold Eq. (11) is set as:

$$
\mathrm{T}(\mathrm{s})= \begin{cases}\frac{\mathrm{p}}{1-\mathrm{p} \cdot\left(\mathrm{r} \bmod \frac{1}{\mathrm{p}}\right)} * \frac{\text { Residual Energy of a node } * \mathrm{k}_{\mathrm{ppt}}}{\text { Average energy of network }} & \text { if } \mathrm{s} \in \mathrm{G} \\ 0 & \text { Otherwise }\end{cases}
$$

The probabilities of normal and advanced nodes in case of twolevel heterogeneity are:

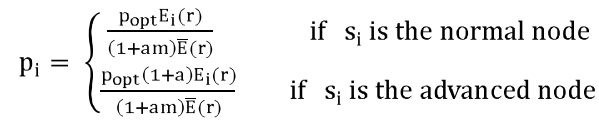

The probabilities of normal, advanced and super nodes in case of two-level heterogeneity are:

$$
p_{i}= \begin{cases}\frac{p_{o p t} E_{i}(r)}{(1+m .(a+m o . b)) \bar{E}(r)} & \text { if } s_{i} \text { is the normal node } \\ \frac{p_{o p t}(1+a) E_{i}(r)}{(1+m \cdot(a+m o \cdot b)) \bar{E}(r)} & \text { if } s_{i} \text { is the advanced node } \\ \frac{p_{o p t}(1+b) E_{i}(r)}{(1+m .(a+m o \cdot b)) \bar{E}(r)} & \text { if } s_{i} \text { is the super node }\end{cases}
$$

The probability of a node to be a cluster head in case of multilevel heterogeneity is given by:

$$
p_{i}=\frac{p_{o p t} \cdot N \cdot\left(1+a_{i}\right) \cdot E_{i}(r)}{\left(N+\sum_{i=1}^{N} a_{i}\right) \bar{E}(r)}
$$

Threshold for cluster head selection is calculated for each type of heterogeneity by putting above values $\mathrm{p}_{\mathrm{i}}$ of Eq. (13), (14) and (15) in Eq. (12).

\section{SIMULATION AND RESULTS}

We have simulated the wireless sensor network in MatLab environment in $100 \times 100$ field. The table 1 shows the simulation parameters used. 
Table 1

Simulation Parameters

\begin{tabular}{|l|l|}
\hline Parameters & Value \\
\hline Network Field & $(100,100)$ \\
\hline Number of nodes & 100 \\
\hline $\begin{array}{l}\text { Eo ( Initial energy of } \\
\text { normal nodes) }\end{array}$ & $0.5 \mathrm{~J}$ \\
\hline Message Size & $4000 \mathrm{Bits}$ \\
\hline $\mathrm{E}_{\text {elec }}$ & $50 \mathrm{~nJ} / \mathrm{bit}$ \\
\hline $\mathrm{E}_{\mathrm{fs}}$ & $10 \mathrm{~nJ} / \mathrm{bit}_{\mathrm{m}}{ }^{2}$ \\
\hline $\mathrm{E}_{\mathrm{amp}}$ & $0.0013 \mathrm{pJ} / \mathrm{bit} / \mathrm{m}^{4}$ \\
\hline $\mathrm{E}_{\mathrm{DA}}$ & $5 \mathrm{~nJ} / \mathrm{bit} / \mathrm{signal}$ \\
\hline $\mathrm{do}($ Threshold Distance) & $70 \mathrm{~m}$ \\
\hline $\mathrm{p}_{\mathrm{opt}}$ & 0.1 \\
\hline
\end{tabular}

We have taken following cases for heterogeneity:

\section{For two-level heterogeneity}

Case 1: $m=0.2, a=3$

Case $2: \mathrm{m}=0.3, \mathrm{a}=1.5$

\section{For three-level heterogeneity}

Case $1: \mathrm{m}=0.5, \mathrm{mo}=0.4, \mathrm{a}=1.5, \mathrm{~b}=3$

Case $2: \mathrm{m}=0.3, \mathrm{mo}=0.6, \mathrm{a}=1, \mathrm{~b}=1.5$

DEEC protocol enhanced for three-level is E-DEEC.

\section{For multilevel heterogeneity}

Energy is taken between $\mathrm{E}_{\mathrm{o}}$ and $\mathrm{a}_{\max }$ where $\mathrm{a}_{\max }$ is 3

\subsection{Two-level Heterogeneity}

\subsubsection{Case 1: $m=0.2, a=3$}

In this case there are 20 advanced nodes deployed with 3 times more energy than normal nodes.

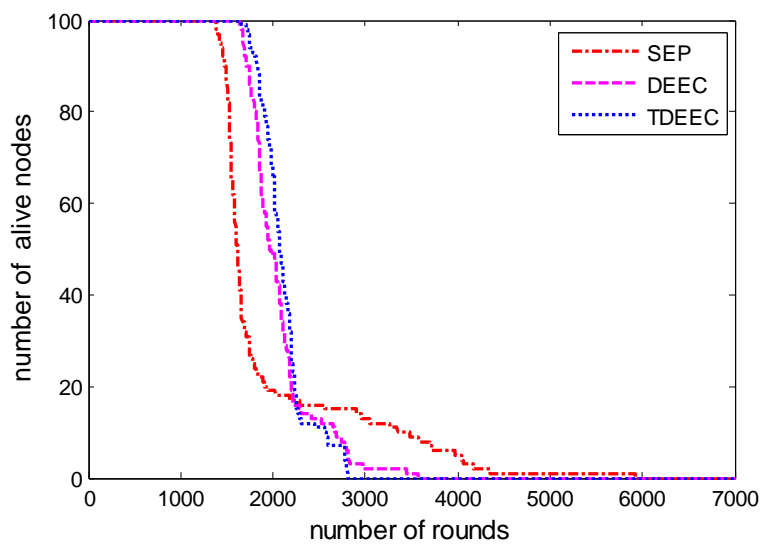

Figure 3 Number of alive nodes over rounds under two-level heterogeneity of SEP, DEEC and TDEEC

From figure 3 we see that stability period and lifetime of TDEEC is more as compared to others. Also, the unstable period of TDEEC is smaller than other protocol as it selects the cluster head based on the ratio of residual energy of node and average energy of the network in respect to the optimum number of cluster heads.

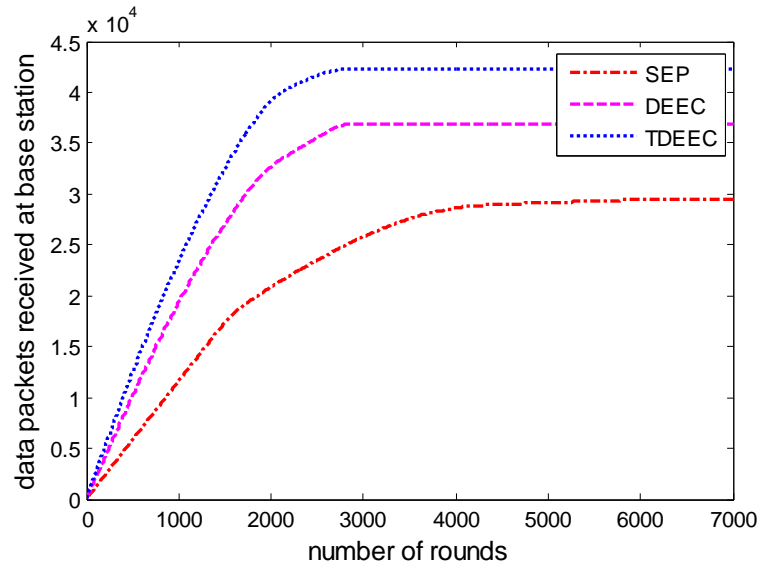

Figure 4 Data Packets over rounds under two-level heterogeneity of SEP, DEEC and TDEEC

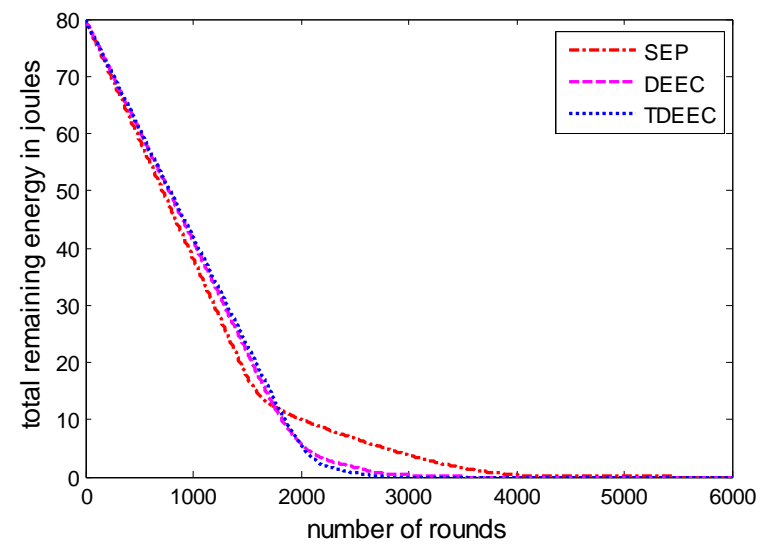

Figure 5 Total remaining energy over rounds under two-level heterogeneity of SEP, DEEC and TDEEC

Also, figure 4 illustrate that data received at the base station per round for TDEEC is more as compared to DEEC and SEP. The results show that for all the protocols it goes linearly for around 2000 rounds and after that the difference can be seen. Figure 5 show that total initial energy of the network is $80 \mathrm{~J}$ which decreases linearly up to 2000 rounds. Energy remaining per round for TDEEC is more as compared to others.

\subsubsection{Case 2: $m=0.3, a=1.5$}

In this case there are 30 advanced nodes deployed with 1.5 times more energy than normal nodes i.e., less energy as compared to previous case 1 .

Figure 6 represent the number of nodes that are alive and dead during the lifetime of the network. In this case also stability period and lifetime of TDEEC is more as compared to others. Also, the unstable period of TDEEC is smaller than other protocol Death of first node of TDEEC starts at around 1600 round and last node dies at around 2200

From figure 7 we can see that data received at the base station per round for TDEEC is more than DEEC and SEP. The results show that for all the protocols it goes linearly for around 2500 rounds and after that the difference can be seen 


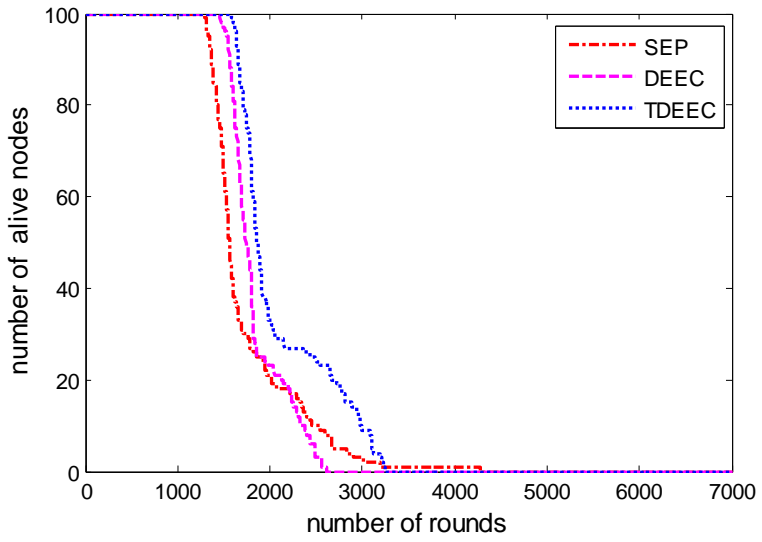

Figure 6 Number of alive nodes over rounds under two-level heterogeneity of SEP, DEEC and TDEEC

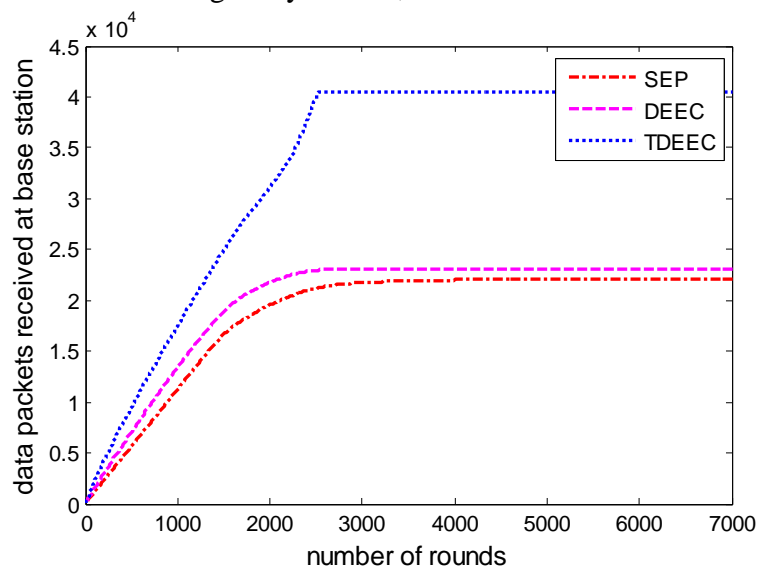

Figure 7 Data Packets over rounds under two-level heterogeneity of SEP, DEEC and TDEEC

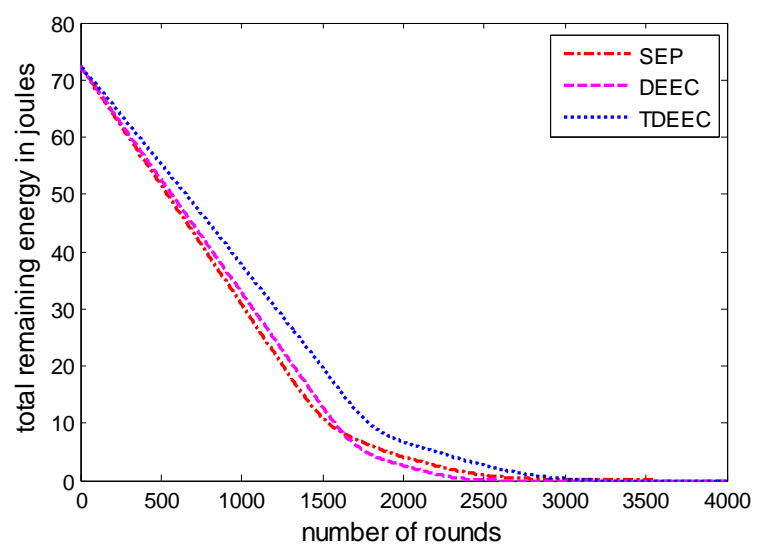

Figure 8 Total remaining energy over rounds under two-level heterogeneity of SEP, DEEC and TDEEC

Figure 8 shows that total initial energy of the network is $70 \mathrm{~J}$ which decreases linearly up to 2500 rounds and after that there is a difference from the round where first node dies in respect to them. Energy remaining per round for TDEEC is more as compared to others.

\subsection{Three-level Heterogeneity}

\subsubsection{Case 1: $m=0.5, m o=0.4, a=1.5, b=3$}

In this case there are 20 advanced nodes deployed with 1.5 times more energy than normal nodes and 30 super nodes deployed with 3 times more energy than the normal nodes. Hence more total initial energy.

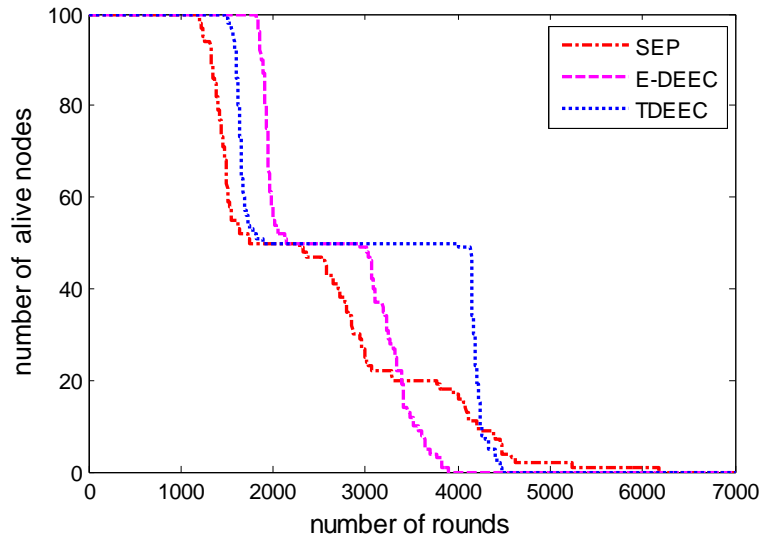

Figure 9 Number of alive nodes over rounds under three-level heterogeneity of SEP, E-DEEC and TDEEC

In this case TDEEC first node dies at around 1500 rounds and last node dies at 4500 . From around round 2000 to 4000 it shows constant number of alive nodes and at that time data received at base station is comparatively more than others. Hence most of the energy is consumed up to 4000 round. Figure 10 shows the comparison in terms of number of data packets received at the base station. The results show that TDEEC goes linearly for around 4000 rounds. It is clear TDEEC has more numbers of data packets send to base station as compared to the SEP and TDEEC.

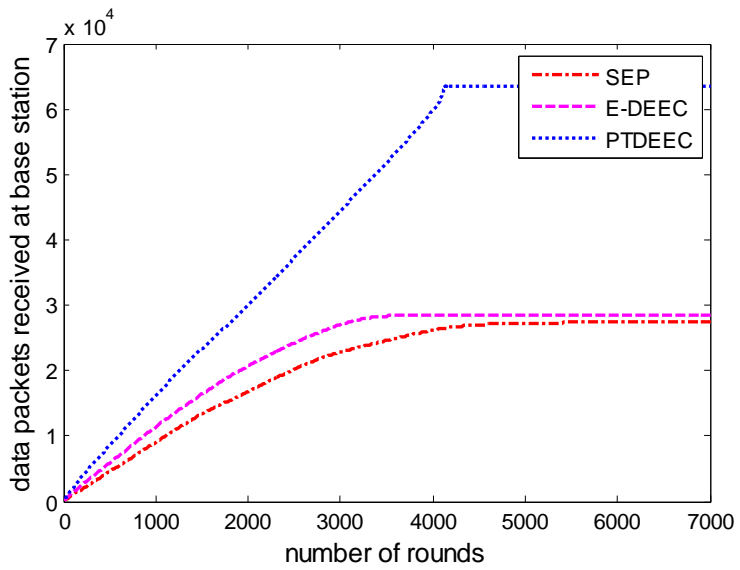

Figure 10 Data Packets over rounds under three-level heterogeneity of SEP, E-DEEC and TDEEC

Figure 11 show total remaining energy over time i.e., number of rounds. Here total initial energy is $102.5 \mathrm{~J}$ which decreases linearly up to around 1500 rounds for both TDEEC. Energy per round is more in TDEEC as compared to others Most of the energy is consumed in the first 4000 rounds for TDEEC. 


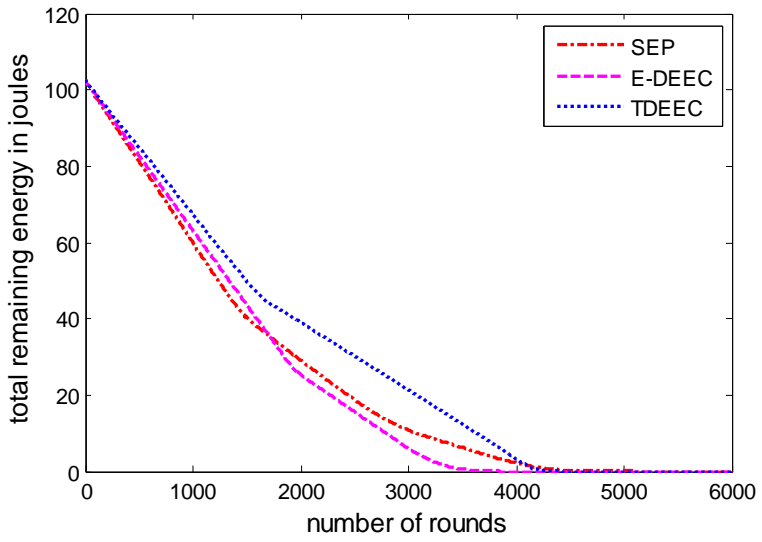

Figure 11 Total remaining energy over rounds under three-level heterogeneity of SEP, E-DEEC and TDEEC

\subsubsection{Case 2: $m=0.3, m o=0.6, a=1, b=1.5$}

In this case, there are 12 advanced nodes deployed with 1 times more energy than normal nodes and 18 super nodes deployed with 1.5 times more energy than the normal nodes.

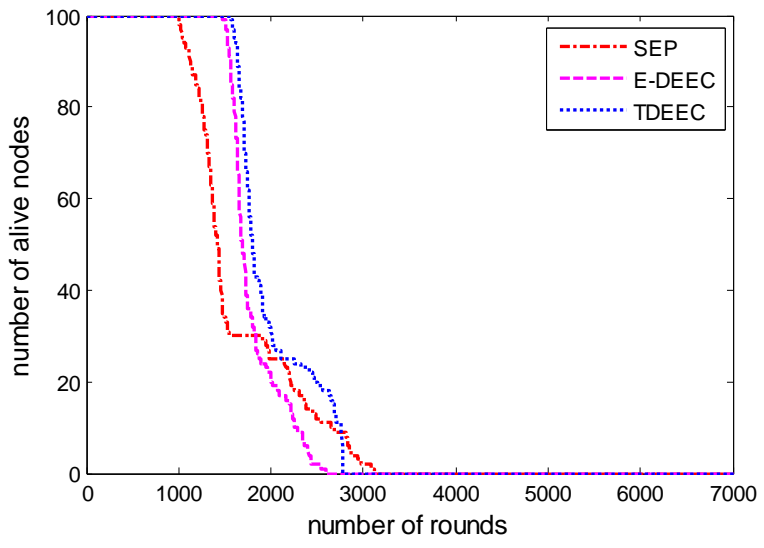

Figure 12 Number of alive nodes over rounds under three-level heterogeneity of SEP, E-DEEC and TDEEC

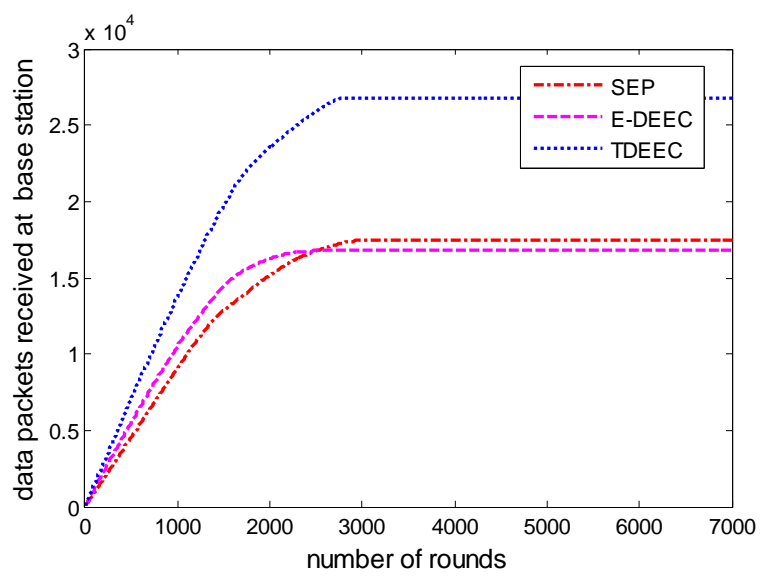

Figure 13 Data Packets over rounds under three-level heterogeneity of SEP, E-DEEC and TDEEC
In this case stability period and lifetime of TDEEC is longer as compared to other protocols and unstable period of TDEEC is shorter than others as it selects the cluster head based on the ratio of residual energy of node and average energy of the network in respect to the optimum number of cluster heads as shown in figure 12. First node of TDEEC dies at 1500 and last node dies at 2700. Figure 13 shows the comparison in terms of number of data packets received at the base station. The results show that TDEEC goes linearly for around 2700 rounds. It is clear from the figure 13 that data that are received by the base station per round is more as compared to others.

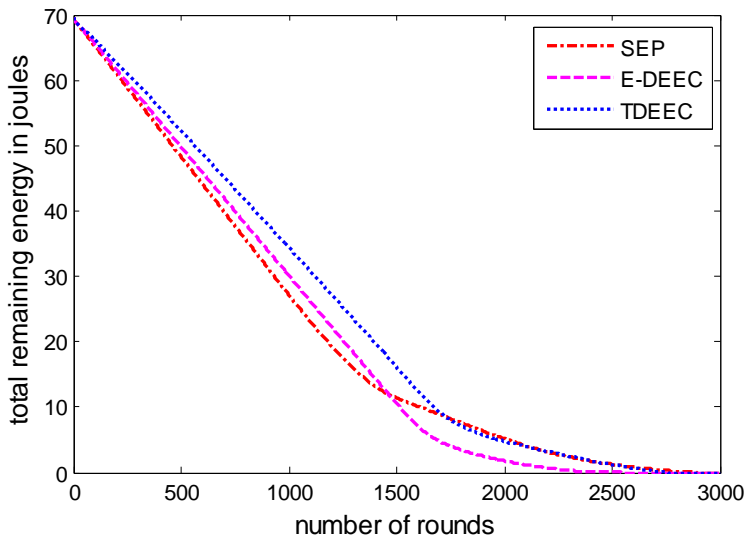

Figure 14 Total remaining energy over rounds under three-level heterogeneity of SEP, E-DEEC and TDEEC

Figure 14 show total remaining energy over time i.e., number of rounds. Here total initial energy is $102.5 \mathrm{~J}$ which decreases linearly up to around 2000 rounds for all the protocols. Energy per round is more in TDEEC as compared to others. Most of the energy is consumed in the first 2700 rounds.

\subsection{Multilevel Heterogeneity}

In this case all the nodes have different initial energy within the set $[0.5,3]$. Here the total initial energy is more as compared to above types of heterogeneity.

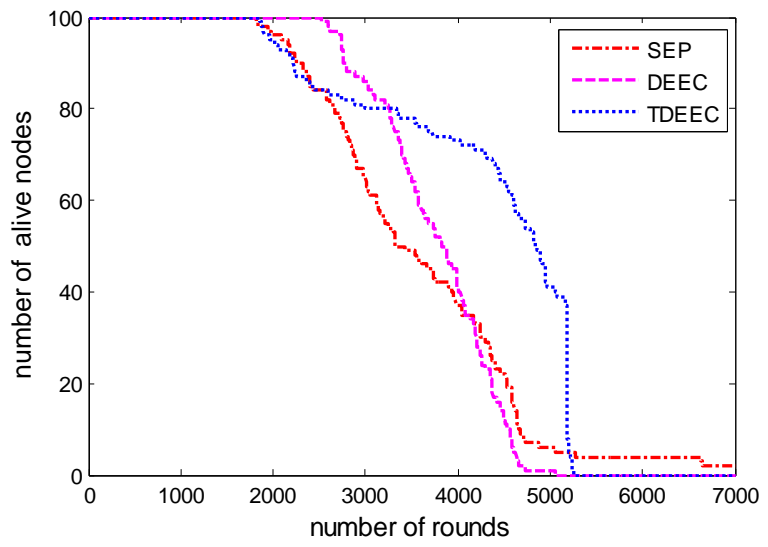

Figure 15 Number of alive nodes over rounds under multilevel heterogeneity of SEP, DEEC and TDEEC

Figure 15 illustrate that first node in TDEEC dies at 1750 round and last node dies around at 5200 round. Between rounds 3300 and 5200 TDEEC performs much better as compared to DEEC 
and SEP. There is vast improvement in terms of data packets send that are received by the base station as shown in figure 16 and therefore most of the energy is consumed in rounds between 2500 and 5000 and then all nodes rapidly as shown in figure 17 .

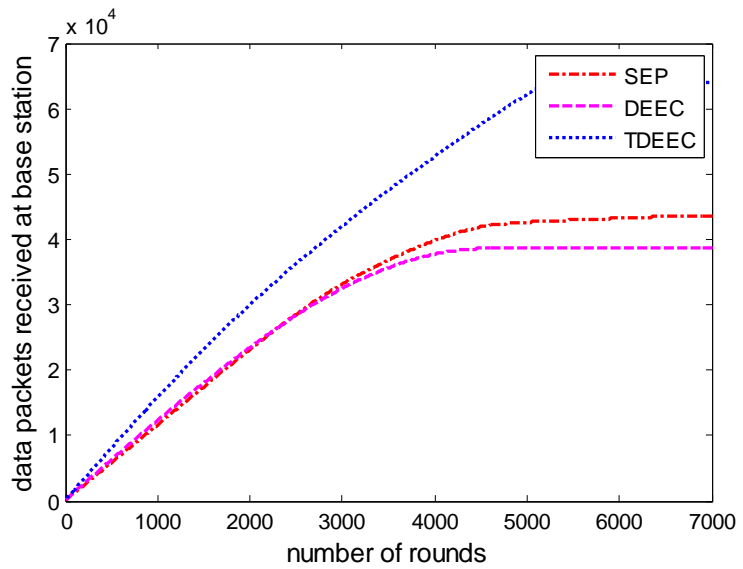

Figure 16 Data Packets over rounds under multilevel heterogeneity of SEP, DEEC and TDEEC

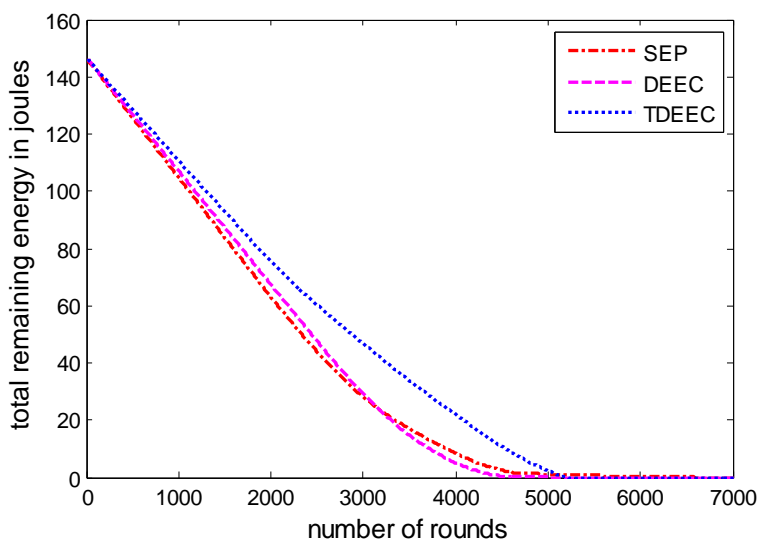

Figure 17 Total remaining energy over rounds under multilevel heterogeneity of SEP, DEEC and TDEEC

\section{CONCLUSION}

In this paper we proposed TDEEC (Threshold Distributed Energy Efficient Clustering) protocol which improves stability and energy efficient property of the heterogeneous wireless sensor network and hence increases the lifetime. Simulation results show that TDEEC performs better as compared to SEP and DEEC in heterogeneous environment for wireless sensor networks.

\section{REFERENCES}

[1] Jamal N. Al-Karaki, Ahmed E. Kamal,", Routing Techniques in Wireless Sensor Networks: A Survey", IEEE Wireless Communications, Volume: 11, Issue: 6 , 26-28, December 2004

[2] W.Heinzelman, A. Chandrakasan, and H. Balakrishnan, "Energy-efficient communication protocol for wireless sensor networks," in the Proceeding of the Hawaii International Conference System Sciences, Hawaii, January 2000

[3] W.R. Heinzelman, A.P. Chandrakasan, H. Balakrishnan, An application specific protocol architecture for wireless microsensor networks, IEEE Transactions on Wireless Communications 1 (4) (2002) 660-670.

[4] O. Younis, S. Fahmy, "HEED: A Hybrid, Energy efficient, Distributed Clustering approach for adhoc sensor networks", IEEE Transactions on mobile computing Vol 3, No 4, pp 660$669,2004$.

[5] G. Smaragdakis, I. Matta, A. Bestavros, SEP: A Stable Election Protocol for clustered heterogeneous wireless sensor networks, in: Second International Workshop on Sensor and Actor Network Protocols and Applications (SANPA 2004), 2004.

[6] Md. Solaiman Ali, Tanay Dey, and Rahul Biswas, "ALEACH: Advanced LEACH Routing Protocol for Wireless Microsensor Networks" ICECE 2008, 20-22 December 2008.

[7] L. Qing, Q. Zhu, M. Wang, "Design of a distributed energyefficient clustering algorithm for heterogeneous wireless sensor networks". ELSEVIER, Computer Communications 29, pp 2230- 2237, 2006.

[8] S. Lindsey and C. S. Raghavendra, "PEGASIS: Power Efficient GAthering in Sensor Informatio Systems," in the Proceedings of the IEEE Aerospace Conference, Big Sky, Montana, March 2002.

[9] A. Manjeshwar and D. P. Agarwal, "TEEN: a routing protocol for enhanced efficiency in wireless sensor networks," In 1st International Workshop on Parallel and Distributed Computing Issues in Wireless Networks and Mobile Computing, April 2001

[10] A. Manjeshwar and D. P. Agarwal, "APTEEN: A hybrid protocol for efficient routing and comprehensive information retrieval in wireless sensor networks," Parallel and Distributed Processing Symposium., Proceedings International, IPDPS 2002, pp. 195-202.

[11] U. Sajjanhar, P. Mitra, "Distributive Energy Efficient Adaptive Clustering Protocol for Wireless Sensor Networks", Proceedings of the 2007 International Conference on Mobile Data Management, pp. 326 - 330, 2007.

[12] Elbhiri Brahim,Saadane Rachid,Alba-Pages Zamora, Driss Aboutajdine, "Stochastic Distributed Energy-Efficient Clustering (SDEEC) for heterogeneous wireless sensor networks", ICGST-CNIR Journal, Volume 9, Issue 2, December 2009.

[13] Inbo Sim, KoungJin Choi, KoungJin Kwon and Jaiyong Lee, "Energy Efficient Cluster header Selection Algorithm in WSN", International Conference on Complex, Intelligent and Software Intensive Systems , IEEE, 2009.

[14] Ma Chaw Mon Thein, Thandar Thein "An Energy Efficient Cluster-Head Selection for Wireless Sensor Networks", International Conference on Intelligent Systems, Modeling and Simulation, IEEE 2009

[15] Dilip Kumar, Trilok C. Aseri, R.B. Patel, "EEHC: Energy efficient heterogeneous clustered scheme for WSNs", ELSEVIER, Computer Communications, 32 (2009) 662-667

[16] Yingchi Mao, Zhen Liu, Lili Zhang, Xiaofang Li, “An Effective Data Gathering Scheme in Heterogeneous Energy WSNs, International Conference on Computational Science and Engineering,2009. 